\title{
PAPR-Limited Precoding in Massive MIMO Systems with Reflect- and Transmit-Array Antennas
}

\author{
Ali Bereyhi*, Vahid Jamali*, Ralf R. Müller*, Georg Fischer*, Robert Schober*, and Antonia M. Tulino ${ }^{\dagger}$ \\ *Friedrich-Alexander Universität Erlangen-Nürnberg, \\ ${ }^{\dagger}$ Nokia Bell Labs and University degli Studi di Napoli Federico II \\ \{ali.bereyhi, vahid.jamali, ralf.r.mueller, georg.fischer, robert.schober\}@fau.de, a.tulino@ nokia-bell-labs.com
}

\begin{abstract}
Conventional hybrid analog-digital architectures for millimeter-wave massive multiple-input multiple-output (MIMO) systems suffer from poor scalability and high implementational costs. The former is caused by the high power loss in the analog network, and the latter is due to the fact that classic MIMO transmission techniques require power amplifiers with high back-offs.

This paper proposes a novel hybrid analog-digital architecture which addresses both of these challenges. This architecture implements the analog front-end via a passive reflect- or transmit-array to resolve the scalability issue. To keep the system cost-efficient, a digital precoder is designed whose peak-to-average power ratio (PAPR) on each active antenna is tunable. Using the approximate message passing algorithm, this precoder is implemented with tractable computational complexity. The proposed architecture allows for the use of power amplifiers with low back-offs which reduces the overall radio frequency cost of the system. Numerical results demonstrate that for low PAPRs, significant performance enhancements are achieved compared to the state of the art.
\end{abstract}

Index Terms-Massive MIMO, mmWave, hybrid analog-digital precoding, regularized least-squares, reflect- and transmit-arrays, approximate message passing.

\section{INTRODUCTION}

Recent studies have shown the necessity of employing massive multiple-input multiple-output (MIMO) settings and moving towards the millimeter-wave (mmWave) spectrum to meet the data rate demands in the next generations of wireless communication systems [1], [2]. This fact has drawn attention to the concept of hybrid analog-digital (HAD) precoding [3], [4]. Conventional HAD transmitters suffer from high power loss in their analog feed networks. As a solution to this issue, an energy-efficient HAD architecture based on reflect-array (RA) and transmit-array (TA) antennas has been recently proposed in [5]. In contrast to conventional designs, this architecture is fully scalable with respect to the number of antennas.

The initial design in [5] employs linear precoding in the digital unit. Despite the advantage of low computational complexity, there is a downside to such precoders: the peak-to-average power ratio (PAPR) at each radio frequency chain $(R F C)$ is not restricted. Noting that the implementation cost scales with the dynamic range 1 of the RFCs, high PAPR either increases

This work has been presented in the 2019 Asilomar Conference on Signals, Systems, and Computers. The link to the final version in the proceedings will be available later.

${ }^{1}$ By dynamic range, we mean the power range in which the power amplifier of the RFC behaves linearly. This range is often quantified by the back-off of the power amplifier. the cost for a desired performance level or causes performance degradation for a fixed budget.

\section{A. Contributions}

In this study, we address the problem of constraining the PAPR of HAD architectures focusing on the recent proposal in [5]. Invoking the generalized least square error (GLSE) scheme introduced in [6]-[8], we design a digital precoder whose output PAPR on each active antenna is tunable. The proposed precoder is implemented via the approximate message passing (AMP) algorithm, so that its computational complexity scales linearly with the number of active antennas. Our results demonstrate that by using the proposed scheme the performance is significantly enhanced for low PAPRs.

\section{B. Notation}

Throughout the paper, scalars, vectors, and matrices are represented by non-bold, bold lower case, and bold upper case letters, respectively. $\mathbf{I}_{K}$ is the $K \times K$ identity matrix, and $\mathbf{H}^{\mathrm{H}}$ is the conjugate transpose of $\mathbf{H}$. $\|\mathbf{H}\|_{F}$ denotes the Frobenius norm of $\mathbf{H} . \mathbb{R}$ and $\mathbb{C}$ are the real axis and complex plane, respectively. $\mathbb{E}\{\cdot\}$ denotes mathematical expectation. For simplicity, $\{1, \ldots, N\}$ is abbreviated by $[N]$.

\section{Problem Formulation}

Consider downlink transmission in a multiuser MIMO system with a base station (BS) and $K$ single-antenna users. The BS employs the RA/TA HAD architecture proposed in [5]. The architecture is shown in Fig. 1 and reviewed below.

\section{A. Transceiver Architecture}

The transmitter consists of a digital signal processing unit with $N$ RFCs and a passive array with $M$ antenna elements. This passive array is either an RA consisting of $M$ passive reflectors or a TA with $M$ passive re-transmitters. The array is located at distance $R_{\mathrm{d}}$ from the RFCs; see Fig. 1.

\section{B. HAD Precoding}

Let $s_{k}$ be the information symbol intended for user $k$. The digital unit maps $s=\left[s_{1}, \ldots, s_{K}\right]^{\top}$ to the transmit signal $\boldsymbol{x} \in \mathbb{C}^{N}$ using the digital precoder $\Pi_{\mathrm{d}}(\cdot): \mathbb{C}^{K} \mapsto \mathbb{C}^{N}$. Hence, the digitally precoded signal is given by

$$
\boldsymbol{x}=\Pi_{\mathrm{d}}(s) .
$$




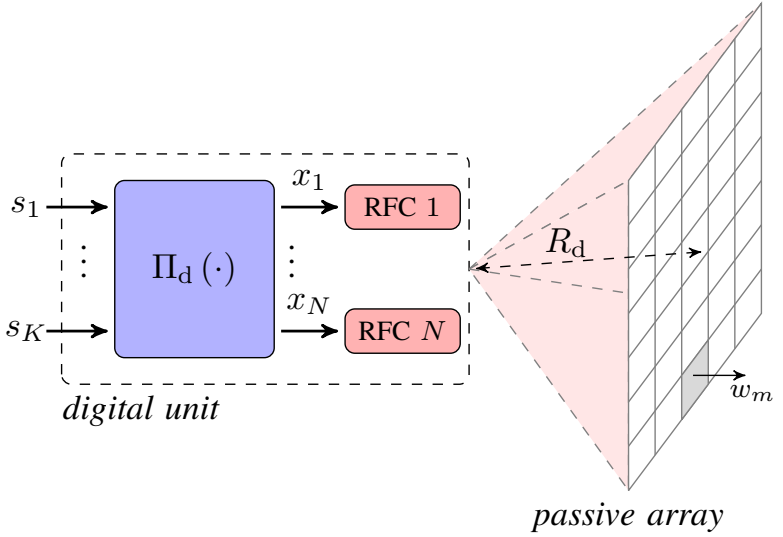

Fig. 1: Block diagram of the RA/TA HAD transceiver.

The transmit signal $\boldsymbol{x}$ is then radiated via the RFCs towards the passive array. Each element of the array either reflects or re-transmits its receive signal after applying a phase shift. Following the characterization in [5], the signal transmitted by the passive array is written as

$$
\boldsymbol{w}=\mathbf{D T} \boldsymbol{x} .
$$

In (2), the matrices $\mathbf{T}$ and $\mathbf{D}$ are defined as follows:

- $\mathbf{T} \in \mathbb{C}^{M \times N}$ models the linear channel from the RFCs to the passive array. In general, $\mathbf{T}$ depends on the array positioning and characteristics. When all the active antennas radiate with the same pattern and the passive elements are isotropic, the $(m, n)$ entry of $\mathbf{T}$ is given by [5]

$$
[\mathbf{T}]_{m n}=\left[\frac{\lambda \sqrt{\zeta G\left(\theta_{m n}, \phi_{m n}\right)}}{4 \pi r_{m n}} \exp \left\{-\mathrm{j} \frac{2 \pi r_{m n}}{\lambda}\right\}\right] \text {, }
$$

for $n \in[N]$ and $m \in[M]$. In (3),

$-\lambda$ is the wavelength.

- $\zeta$ is the power efficiency of the passive antenna array.

- $G(\theta, \phi)$ denotes the radiation pattern of the active antennas where $\theta$ and $\phi$ are the elevation and azimuth angles, respectively.

- $\left(r_{m n}, \theta_{m n}, \phi_{m n}\right)$ is the relative spherical coordinate of the $m$-th passive element when the origin is located at the $n$-th active antenna.

- $\mathbf{D}$ is an $M \times M$ diagonal matrix which models the phase shifts applied by the passive antenna elements. The diagonal entries of $\mathbf{D}$ are given by

$$
[\mathbf{D}]_{m m}=\exp \left\{\mathrm{j} 2 \pi \beta_{m}\right\},
$$

where $0 \leq \beta_{m} \leq 1$. In practice, the passive elements apply quantized phase shifts; for example, architectures implemented by transmission lines [9], [10]. Hence, $\beta_{m}$ takes values from some set $\mathbb{B}=\left\{B_{1}, \ldots, B_{Q}\right\} \subset[0,1]$, where $Q$ denotes the number of quantization levels.

\section{Channel Model}

The signal radiated by the passive array is transmitted over a Gaussian broadcast channel which experiences quasi-static fading. The matrix of channel gains is denoted by $\mathbf{H} \in \mathbb{C}^{K \times M}$ and is known at the BS prior to signal transmission. The vector of receive signals, i.e., $\boldsymbol{y}=\left[y_{1}, \ldots, y_{K}\right]^{\top}$ is hence given by

$$
\boldsymbol{y}=\mathbf{H} \boldsymbol{w}+\boldsymbol{z}=\mathbf{H D T} \boldsymbol{x}+\boldsymbol{z},
$$

where $\boldsymbol{z}$ is additive white Gaussian noise with zero mean and variance $\sigma^{2}$, i.e., $\boldsymbol{z} \sim \mathcal{C N}\left(0, \sigma^{2} \mathbf{I}_{K}\right)$.

\section{Main Objectives}

The HAD transmitter involves $\mathbf{T}, \mathbf{D}$ and $\Pi_{\mathrm{d}}(\cdot)$, which need to be designed:

(a) The design of $\mathbf{T}$ mainly requires the tuning of the antenna characteristics and the array position.

(b) For the design of $\mathbf{D}$, the optimal phase shift of each antenna element should be determined.

(c) The digital precoder $\Pi_{\mathrm{d}}(\cdot)$, which is implemented in the baseband domain, needs to be designed for a given set of signal constraints, e.g. limited transmit power.

The update rates of these three blocks are not the same. D and $\Pi_{\mathrm{d}}(\cdot)$ are often updated multiple times within a coherence time interval while $\mathbf{T}$ is designed offline. We hence assume that $\mathbf{T}$ has been tuned in advance and is kept fixed afterwards.

Existing proposals consider linear digital precoders [5], i.e., $\Pi_{\mathrm{d}}(\boldsymbol{s})=\mathbf{A} \boldsymbol{s}$ for some precoding matrix $\mathbf{A}$. Such schemes can result in high PAPR at the RFCs. To address this issue, in this paper, we invoke the GLSE framework, recently introduced in [6]-[8], and propose a PAPR-limited digital precoder based on the regularized least-squares (RLS) method. For the sake of tractability, we further develop an iterative algorithm based on AMP to implement the digital precoder. The complexity of this algorithm scales linearly with the number of active antenna elements. This makes the proposed scheme practically feasible for massive MIMO settings. The tuning strategy for D is further discussed briefly in Section III-B

\section{E. Performance Metric}

To quantify the performance, we consider the residual sum of squares (RSS) at the receiver side as the metric. For a given vector of information symbols $s$ and its corresponding digitally precoded signal $\boldsymbol{x}=\Pi_{\mathrm{d}}(\boldsymbol{s})$, the RSS is defined as

$$
\operatorname{RSS}(\boldsymbol{x}, \mathbf{D} \mid \mathbf{T})=\|\mathbf{H D T} \boldsymbol{x}-\boldsymbol{s}\|^{2} .
$$

The RSS quantifies the average distortion between the noisefree receive signals and the information symbols. In the ideal case, where the end-to-end channel is inverted, the RSS is zero.

\section{HAD PRECODING WITH MINIMUM RSS}

For given $s, \mathbf{T}$, and signal constraints, the optimal choices of $\mathbf{D}$ and $\Pi_{\mathrm{d}}(\cdot)$ with respect to the RSS are

$$
\left(\Pi_{\mathrm{d}}(\boldsymbol{s}), \mathbf{D}\right)=\underset{\tilde{\Pi} \in \mathcal{P}, \tilde{\mathbf{D}} \in \mathcal{D}}{\operatorname{argmin}} \operatorname{RSS}(\tilde{\Pi}(s), \tilde{\mathbf{D}} \mid \mathbf{T}),
$$

\footnotetext{
${ }^{2}$ More generally, the RSS can be defined as the distance between the noisefree receive signals and a scaled version of the information symbols, i.e., replacing $s$ with $\alpha s$ for some scalar $\alpha$. For sake of simplicity, we set $\alpha=1$.
} 
where $\mathcal{P}$ is the set of all mappings whose output entries satisfy the given signal constraints. For example, if the transmit signal is restricted to have a limited peak power, $\mathcal{P}$ contains all functions $\tilde{\Pi}(\cdot): \mathbb{C}^{K} \mapsto \mathbb{C}^{N}$ for which the entries of the precoded signal $\tilde{\boldsymbol{x}}=\tilde{\Pi}(s)$ satisfy $\left|\tilde{x}_{n}\right|^{2} \leq P$ for some $P$. Moreover, set $\mathcal{D}$ contains all possible phase shift matrices.

In (7), $\mathbf{T}$ is specified by the position and characteristics of the passive and active antenna arrays. In this respect, one can design the system such that

$$
\mathbf{T}=\underset{\tilde{\mathbf{T}} \in \mathcal{T}}{\operatorname{argmin}} \mathbb{E}_{\mathbf{H}, \boldsymbol{s}}\left\{\operatorname{RSS}\left(\Pi_{\mathrm{d}}(\boldsymbol{s}), \mathbf{D} \mid \tilde{\mathbf{T}}\right)\right\}
$$

where $\mathcal{T}$ is the set of all possible channel matrices between the active and passive array $\$ 3$. The expectation in $(8)$ averages the RSS over all realizations of the channel coefficients, i.e., $\mathbf{H}$, and the information symbols.

In the sequel, we focus on the design of the digital precoder and the passive phase shifters considering a limited PAPR as the design constraint. To this end, we note that

- The RSS is in general a mixed function of $\Pi_{d}(\cdot)$ and $\mathbf{D}$ meaning that (7) cannot be decomposed into two decoupled optimization sub-problems in terms of $\Pi_{\mathrm{d}}(\cdot)$ and $\mathbf{D}$.

- The diagonal entries of $\mathbf{D}$ do not take values from a convex set. Hence, the global minimum of the optimization problem in (7) is not tractable.

To address these issues, we decompose the problem in (7) into two mutually coupled sub-problems. In the first sub-problem, we find the optimal precoder as a function of $\mathbf{D}$ via the RLS method. Then, we substitute the solution into (7) and find an approximation of the optimal choice for matrix $\mathbf{D}$, noting that this latter task is non-deterministic polynomial-time (NP)-hard. We discuss our approach in detail in the following sections.

\section{A. Designing the Digital Unit}

Assuming a fixed $\mathbf{D}$, one can interprete the effective end-toend channel $\mathbf{H}_{\mathrm{e}}=\mathbf{H D T}$ as a matrix of $N$ regressors, and $s$ as a vector of $K$ regressands assumed to be linearly related to the regressors via $N$ regression coefficients. These coefficients are the entries of the digitally precoded signal $\boldsymbol{x}$. By this interpretation, RSS minimization is mathematically equivalent to the least-squares formulation of this linear regression problem 4 .

The standard approach to solving this equivalent regression problem is to utilize the RLS method in which we minimize a penalized version of the RSS. The penalty term, often referred to as the regularization term, is proportional to the constraints required to be satisfied by the coefficients. Considering the particular constraint of PAPR limitation, we note the following items:

1) The average power of the transmit signal is required to be restricted. Such a constraint can be imposed by penalizing the RSS with a term proportional to $\|\boldsymbol{x}\|^{2}$.

2) The power of the entries of $\boldsymbol{x}$, i.e., $x_{n}$ for $n \in[N]$, should be bounded from above. Such a constraint is enforced by

\footnotetext{
${ }^{3}$ Note that in $(8)$, the digital precoder and the matrix of phase shifts in the objective function are given by $(7)$ which are also functions of $\tilde{\mathbf{T}}$.

${ }^{4}$ See [8] for more details on this representation.
}

adding a barrier function to the RSS which tends to infinity as $\left|x_{n}\right|^{2}>P$ for some peak power $P$. Alternatively, we can restrict the support of the entries of $\boldsymbol{x}$, over which we minimize the RSS, to

$$
\mathbb{X}=\left\{x \in \mathbb{C}:|x|^{2} \leq P\right\} .
$$

Considering the above discussion, the RLS-based digital precoder with limited PAPR is given by

$$
\Pi_{\mathrm{d}}(\boldsymbol{s} \mid \mathbf{D}, \mathbf{T}, \mathbf{H})=\underset{\boldsymbol{v} \in \mathbb{X}^{N}}{\operatorname{argmin}}\|\mathbf{H D T} \boldsymbol{v}-\boldsymbol{s}\|^{2}+\mu_{\mathrm{rls}}\|\boldsymbol{v}\|^{2}
$$

with $\mathbb{X}$ given in (9) and some scalar $\mu_{\mathrm{rls}}$. We refer to $\mu_{\mathrm{rls}}$ as the regularizer. The arguments $\mathbf{D}, \mathbf{T}$, and $\mathbf{H}$ further indicate the dependency of $\Pi_{d}(\cdot)$ on the realizations of the channel coefficients and analog modules.

The precoder in (10) describes a state-dependent GLSE precoder [8] whose output is calculated in polynomial time via convex optimization techniques. However, one can further reduce the computational complexity via AMP. In [11], a class of iterative algorithms based on AMP has been developed for generic GLSE precoding schemes. Invoking the formulation in [11], the precoding scheme in (10) is implemented iteratively, such that its complexity grows linearly with $N$. For the sake of brevity, we skip the derivations for the AMP-based algorithm and refer interested readers to [11] for more details.

\section{B. Designing the Phase Shifters}

The digital precoder in (10) is designed as a function of $\mathbf{D}$. The phase shifts for the passive antenna elements are hence optimally set by minimizing the RSS with respect to D. In other words, $\mathbf{D}$ is optimally tuned by solving

$$
\mathbf{D}=\underset{\tilde{\mathbf{D}} \in \mathcal{D}}{\operatorname{argmin}} \operatorname{RSS}\left(\Pi_{\mathrm{d}}(s \mid \tilde{\mathbf{D}}, \mathbf{T}, \mathbf{H}), \tilde{\mathbf{D}} \mid \mathbf{T}\right) .
$$

$\mathbf{D}$ can be written as $\mathbf{D}=\operatorname{diag}\{\mathbf{d}\}$ for some vector $\mathbf{d} \in \mathbb{D}^{M}$, where 5

$$
\mathbb{D}=\left\{\exp \left\{\mathrm{j} 2 \pi B_{1}\right\}, \ldots, \exp \left\{\mathrm{j} 2 \pi B_{Q}\right\}\right\}
$$

with $0 \leq B_{q} \leq 1$ for $q \in[Q]$. As a result, the optimal choice for $\mathbf{D}$ is written as $\mathbf{D}=\operatorname{diag}\left\{\mathbf{d}^{\star}\right\}$ where

$$
\mathbf{d}^{\star}=\underset{\mathbf{d} \in \mathbb{D}^{M}}{\operatorname{argmin}} f(\mathbf{d})
$$

with

$$
f(\mathbf{d})=\operatorname{RSS}\left(\Pi_{\mathrm{d}}(\boldsymbol{s} \mid \operatorname{diag}\{\mathbf{d}\}, \mathbf{T}, \mathbf{H}), \operatorname{diag}\{\mathbf{d}\} \mid \mathbf{T}\right) .
$$

The direct approach to solving (13) is not tractable for the following reasons:

1) Since the precoded signal is a function of $\mathbf{D}, f(\mathbf{d})$ does not have a simple analytical form.

2) Even for convex forms of $f(\mathbf{d})$, the optimization problem in (13) reduces to the problem of integer programming, and hence is an NP-hard problem 6 .

\footnotetext{
${ }^{5}$ In the limiting case of $Q \rightarrow \infty, \mathbb{D}$ converges to the unit circle

${ }^{6}$ Note that the problem remains NP-hard, even when $Q \rightarrow \infty$.
} 


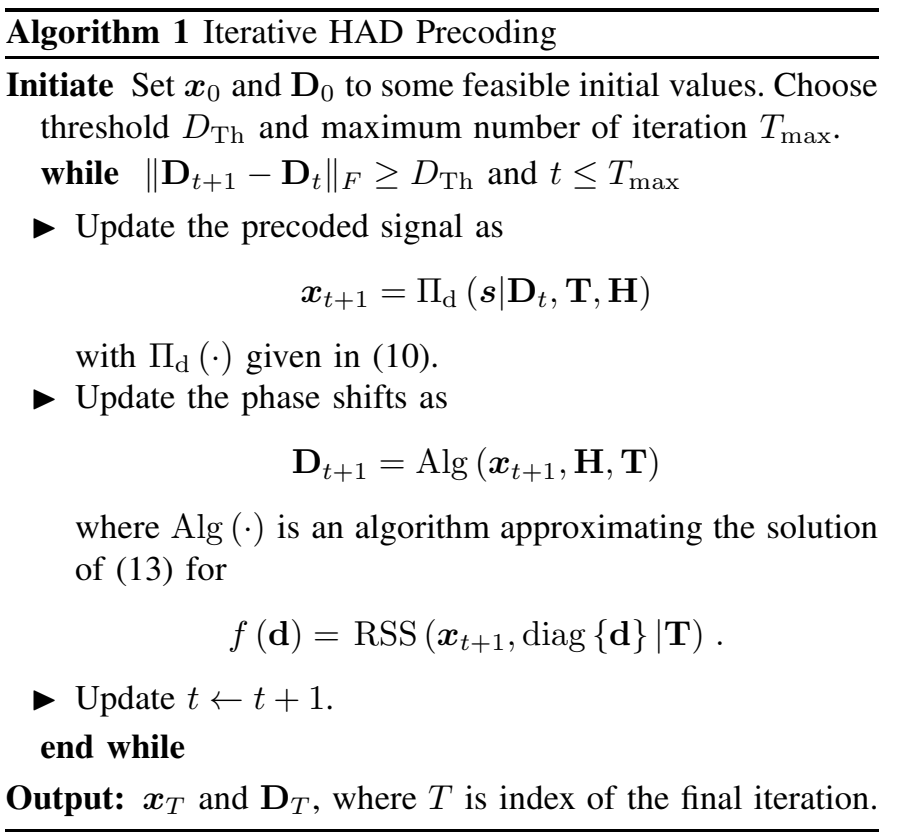

We hence develop a suboptimal approach which approximates the solution of (13). This approach finds $\mathbf{D}$ and its corresponding precoded signal iteratively. In each iteration,

- the precoded signal $\boldsymbol{x}$ is first determined for a fixed $\mathbf{D}$ from (10);

- $\mathbf{D}$ is then updated by (13) treating $\boldsymbol{x}$ as a constant vector. Note that for a constant $\boldsymbol{x}, f(\mathbf{d})$ has a quadratic form.

These steps are then repeated with the updated version of $\mathbf{D}$. The algorithm continues iterating until it either fulfills a stop criterion or exceeds the maximum number of iterations. Optimization problem (13) is solved in each iteration via a suboptimal computationally tractable algorithm. We refer to this algorithm as $\mathrm{Alg}(\cdot)$; an example of $\mathrm{Alg}(\cdot)$ can be derived via the iterative gradient projection technique [12] or manifold optimization [13]. For more examples of such algorithms; see [5], [14], [15], and the references therein.

The proposed approach with an exemplary stop criterion is summarized in Algorithm 1

\section{Numerical Results}

To investigate the performance of the proposed scheme, we study a scenario with $K=8$ users, $N=4$ RFCs, and $M=64$ passive elements. Throughout the simulations, the wavelength is set to $\lambda=5 \mathrm{~mm}$.

\section{A. Settings for the Passive and Active Arrays}

The passive array contains 64 antenna elements installed on an aperture of size $4 \lambda \times 4 \lambda$. The active antennas are further arranged on a ring with radius $R_{\mathrm{r}}=\lambda$ parallel to the passive array. The center of the ring and the passive array coincide on the horizontal plane and are separated by $R_{\mathrm{d}}=4 \lambda / \sqrt{\pi}$.
The active antennas on the RFCs radiate with the same pattern which is horizontally omnidirectional and vertically uniform over $[\pi / 6,5 \pi / 6]$. This means that

$$
G(\theta, \phi)=\left\{\begin{array}{ll}
1 & \theta \in[\pi / 6,5 \pi / 6] \text { and } \phi \in[0,2 \pi] \\
0 & \text { otherwise }
\end{array} .\right.
$$

\section{B. Channel, Information Symbols, and Transform $\mathbf{T}$}

The entries of $\mathbf{H}$, as well as the information symbols, are generated independently, where $[\mathbf{H}]_{k m} \sim \mathcal{C N}(0,1 / M)$ and $s_{k} \sim \mathcal{C N}(0,1)$ for $k \in[K]$ and $m \in[M]$. T is first calculated from (3), and then normalized, such that all the entries lie on the unit circle.

\section{Numerical Simulations}

Since our focus is on PAPR restriction at the digital unit, we set $\mathbf{D}$ to a fixed matrix and do not iterate further to optimize $\mathbf{D}$. The phase shifts at the passive array can be further optimized by setting $\operatorname{Alg}(\cdot)$ in Algorithm 11 to the gradient projection scheme given in [12, Algorithm 1].

The simulations are given for $J=10^{4}$ independent realizations. The precoder in (10) is further implemented via AMP following the derivations in [11]. To quantify the performance of the proposed scheme, we define two parameters:

1) The per-antenna PAPR which for active antenna $n$ reads

$$
\operatorname{PAPR}_{n}=\left(\frac{1}{J} \sum_{j=1}^{J}\left|x_{n}(j)\right|^{2}\right)^{-1} \max _{j \in[J]}\left|x_{n}(j)\right|^{2},
$$

where $\boldsymbol{x}(j)$ is the $j$-th realization of the transmit vector. The PAPR is determined by averaging $\mathrm{PAPR}_{n}$ over $n$.

2) The average RSS is defined as

$$
\overline{\operatorname{RSS}}=\frac{1}{K} \sum_{j=1}^{J} \operatorname{RSS}(\boldsymbol{x}(j), \mathbf{D}, \mathbf{T}) .
$$

Fig. 2 shows $\overline{\mathrm{RSS}}$ versus PAPR for the proposed scheme when the peak power is set to $P=1$. The simulation points are found by sweeping the regularizer $\mu_{\mathrm{rls}}$, and the dashed line is obtained by interpolating the simulation points. From Fig. 2, it is observed that as PAPR grows, $\overline{\mathrm{RSS}}$ decreases.

For sake of comparison, we further plot the curve for the regularized zero forcing (RZF) scheme. With RZF, the digital precoder reads

$$
\tilde{\boldsymbol{x}}=\mathbf{H}_{\mathrm{e}}^{\mathrm{H}}\left(\mathbf{H}_{\mathrm{e}} \mathbf{H}_{\mathrm{e}}^{\mathrm{H}}+\mu_{\mathrm{rzf}} \mathbf{I}_{K}\right)^{-1} \boldsymbol{s}
$$

with $\mathbf{H}_{\mathrm{e}}=\mathbf{H D T}$ for some scalar $\mu_{\mathrm{rzf}}$. To restrict the peak power, the RZF signal is clipped prior to transmission, i.e.,

$$
x_{n}=\left\{\begin{array}{ll}
\tilde{x}_{n} & \left|\tilde{x}_{n}\right|^{2}<P \\
\frac{\sqrt{P} \tilde{x}_{n}}{\left|\tilde{x}_{n}\right|} & \left|\tilde{x}_{n}\right|^{2}>P
\end{array},\right.
$$

where $x_{n}$ denotes the entry transmitted by the $n$-th RFC.

\footnotetext{
${ }^{7}$ This radiation pattern is assumed for sake of simplicity. In practice, patterns with high directivity towards the passive array can be used in order to avoid unwanted power loss in the system.
} 


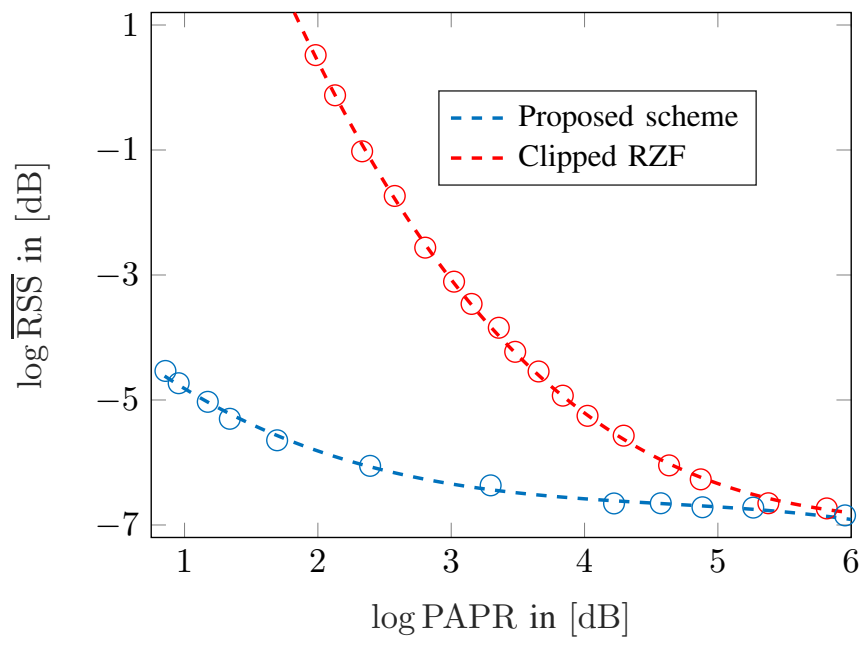

Fig. 2: Average RSS versus average PAPR.

As the figure reveals, at low PAPRs, the proposed scheme significantly outperforms RZF precoding. This is due to the fact that in this regime, the RZF signal is significantly distorted by clipping. For high PAPR, both precoders perform similarly. This observation agrees with the fact that PAPR-limited GLSE precoding reduces to RZF as PAPR $\rightarrow \infty$; see [8].

\section{Conclusions and Final Remarks}

The HAD structure proposed in [5] combined with GLSE precoding [8] results in a cost-efficient transmitter for massive MIMO systems. Utilizing AMP, the preprocessing complexity grows linearly with the number active transmit antennas which is computationally tractable.

The framework presented in this paper is also applicable for other constraints on the transmit signal. Moreover, investigations under realistic millimeter wave channel models further enlighten the efficiency of the proposed scheme. The work in these directions is currently ongoing.

\section{REFERENCES}

[1] T. S. Rappaport, S. Sun, R. Mayzus, H. Zhao, Y. Azar, K. Wang, G. N Wong, J. K. Schulz, M. Samimi, and F. Gutierrez, "Millimeter wave mobile communications for $5 \mathrm{G}$ cellular: It will work!" IEEE access, vol. 1, pp. 335-349, May 2013.

[2] A. V. Delgado, M. Sanchez-Fernandez, J. Llorca, and A. Tulino, "Feasible transmission strategies for downlink MIMO in sparse millimeterwave channels," IEEE Communications Magazine, vol. 56, no. 7, pp. 49-55, July 2018.

[3] O. El Ayach, S. Rajagopal, S. Abu-Surra, Z. Pi, and R. W. Heath, "Spatially sparse precoding in millimeter wave MIMO systems," IEEE Transactions on Wireless Communications, vol. 13, no. 3, pp. 14991513, March 2014.

[4] A. F. Molisch, V. V. Ratnam, S. Han, Z. Li, S. L. H. Nguyen, L. Li, and K. Haneda, "Hybrid beamforming for massive MIMO: A survey," IEEE Communications Magazine, vol. 55, no. 9, pp. 134-141, September 2017.

[5] V. Jamali, A. M. Tulino, G. Fischer, R. R. Müller, and R. Schober, "Scalable and energy-efficient millimeter massive MIMO architectures: Reflect-array and transmit-array antennas," Proc. IEEE International Conference on Communications (ICC), May 2019, Shanghai, China.

[6] A. Bereyhi, M. A. Sedaghat, S. Asaad, and R. Müller, "Nonlinear precoders for massive MIMO systems with general constraints," Proc. International ITG Workshop on Smart Antennas (WSA), March 2017, Berlin, Germany.
[7] A. Bereyhi, M. A. Sedaghat, and R. Müller, "Asymptotics of nonlinear LSE precoders with applications to transmit antenna selection," Proc. International Symposium on Information Theory (ISIT), June 2017, Aachen, Germany.

[8] A. Bereyhi, M. A. Sedaghat, R. R. Müller, and G. Fischer, "GLSE precoders for massive MIMO systems: Analysis and applications," IEEE Transactions on Wireless Communications, vol. 18, no. 9, pp. 44504465, September 2019.

[9] H.-T. Chou, Y.-J. Chen, and H.-K. Ho, "An all-metallic reflectarray and its element design: Exploring the radiation characteristics of antennas for directional beam applications," IEEE Antennas and Propagation Magazine, no. 99, pp. 1-1, October 2018.

[10] A. H. Abdelrahman, F. Yang, A. Z. Elsherbeni, and P. Nayeri, "Analysis and design of transmitarray antennas," Synthesis Lectures on Antennas, vol. 6, no. 1, pp. 1-175, 2017.

[11] A. Bereyhi, M. A. Sedaghat, and R. R. Müller, "Precoding via approximate message passing with instantaneous signal constraints," Proc. International Zurich Seminar on Information and Communication (IZS), pp. 128-132, February 2018, Zürich, Switzerland.

[12] J. Tranter, N. D. Sidiropoulos, X. Fu, and A. Swami, "Fast unit-modulus least squares with applications in beamforming," IEEE Transactions on Signal Processing, vol. 65, no. 11, pp. 2875-2887, June 2017.

[13] P.-A. Absil, R. Mahony, and R. Sepulchre, Optimization algorithms on matrix manifolds. Princeton University Press, USA, 2009.

[14] X. Yu, J.-C. Shen, J. Zhang, and K. B. Letaief, "Alternating minimization algorithms for hybrid precoding in millimeter wave MIMO systems," IEEE Journal of Selected Topics in Signal Processing, vol. 10, no. 3, pp. 485-500, April 2016.

[15] S. S. Ioushua and Y. C. Eldar, "A family of hybrid analog-digital beamforming methods for massive MIMO systems," IEEE Transactions on Signal Processing, vol. 67, no. 12, pp. 3243-3257, June 2019. 